\title{
DORSAL ROOT GANGLIA DEVELOPMENT IN CHICKS FOLLOWING PARTIAL ABLATION OF THE NEURAL CREST ${ }^{1}$
}

\author{
VIRGINIA MCMILLAN CARR
}

Department of Biochemistry, Molecular and Cell Biology, Northwestern University, Evanston, Illinois 60201

Received August 5, 1983; Revised March 22, 1984; Accepted April 17, 1984

\begin{abstract}
To assess the effects of reduced competition for peripheral targets on developing brachial dorsal root ganglia (DRG), chick embryos were subjected to partial ablations of the brachial neural crest at stages 13 or 14 (Hamburger, V., and H. L. Hamilton (1951) J. Morphol. 88: 49-92), using an ophthalmological cauterization unit. In the initial studies reported here, ganglia developing from the remaining crest material were examined for ganglionic volume and neuronal size, neuronal number, and degenerative activity at stage 35 . Results showed that the lesion procedure resulted in the reduction or absence of one or two ganglia on each side at the level of DRG 15 to DRG 17. Hypertrophies occurred in other ganglia remaining at these and at more rostral levels and ranged up to $220 \%$. These hypertrophies were most pronounced, however, not in the ganglia adjacent to those lesioned but rather in more remote ganglia, including those at cervical levels. Accompanying these ganglionic changes were significant alterations in all three neuronal parameters examined. The findings clearly demonstrate a responsiveness of chick brachial DRG to reduced competition resulting from neural crest ablations and that such responsiveness occurs along several axial segments.
\end{abstract}

Previous studies carried out in this laboratory examined the development of the brachial dorsal root ganglia (DRG) in both the normal chick and following early wing bud amputation (Carr and Simpson, 1978a, b). Those studies defined the birthdates of neuronal and non-neuronal cells within these ganglia. They also characterized the proliferative and degenerative events in early ganglionic development in both normal embryos and those subjected to the amputations. Results showed that the initial responses to such a reduction in the peripheral field consisted of greatly elevated degenerative activity followed by a subsequent reduction in glial proliferation. Further analysis of this development, examining the responses of these ganglia to enlargement of their peripheral fields, was also attempted by addition of supernumerary limb buds. However, efforts to obtain well innervated supernumerary limbs located at the brachial level were unsuccessful. This may be a reflection of some unrecognized characteristic of the brachial region (V. Hamburger, personal communication), as successful innervation of supernumerary limbs has been achieved at both the lumbosacral and thoracic levels (Hamburger, 1939a; Hamburger and Levi-Montalcini, 1949; Hollyday and Hamburger,

\footnotetext{
${ }^{1}$ This work was supported by Biomedical Sciences Support Grant 5 S05 RR07028 from the National Institutes of Health to Northwestern University. I wish to thank Dr. Sidney B. Simpson, Jr., for many fruitful discussions as well as his generous donations of research space and supplies. I am also grateful to Drs. S. Simpson and J. Culotti for reviewing this manuscript and to Drs. M. Constantine-Paton and S. Scott for sending me copies of their manuscripts prior to publication.
}

1976; Morris, 1978). However, continued investigation at the brachial level was desirable for several reasons. First, most of the recent quantitative studies of DRG neuronal development in the chick have been carried out at that level so this development is well defined for both normal and various experimental conditions (Carr and Simpson, 1978a, b, 1982; Hamburger et al., 1981). Second, the smaller number of brachial ganglia makes thorough investigation of various ganglionic and cellular parameters much more feasible at the brachial than at the lumbosacral level. Third, recent investigations have demonstrated pharmacological differences in the footshock responses evoked from forelimbs versus hindlimbs of rats (Watkins et al., 1984). Such differences could have possible developmental correlates. Thus, efforts were redirected from limb bud addition to partial ablation of the brachial neural crest. This procedure causes a deletion of DRG precursor cells prior to their migration and condensation into the ganglia. Such a deletion results in a reduced number of neurons confronting a normal peripheral field, a situation not unlike increasing the size of the peripheral field of normal DRG by addition of supernumerary limbs. Similar studies, involving removal of a dorsal quandrant of the early spinal cord along with its accompanying neural crest material, were carried out a number of years ago by Castro (1963). He reported hypertrophy of remaining ganglia but did not investigate possible underlying cellular changes.

The present paper presents the findings of initial studies of DRG development following partial neural crest deletions. In these studies chick embryos were examined at stage 35 (Hamburger and Hamilton, 1951). Parameters examined included ganglionic and neuronal size, neuronal cell numbers, and de- 
generative activity. The results show that by stage 35 major changes occur in each of these parameters.

\section{Materials and Methods}

White Leghorn chick embryos (L. Sharp Co., Glen Ellyn, IL) were incubated at 38 to $39^{\circ} \mathrm{C}$ in a forced draft incubator. At stages 13 and 14 ( 2 days of incubation) the embryos were subjected to neural crest lesions, following which they were reincubated until stage 35 (9 days of incubation). Stage 35 chicks were used in this study because by this stage rapid neuronal growth is occurring (Levi-Montalcini and Levi, 1943) while degenerative activity is still pronounced (Carr and Simpson, 1978a, b; Hamburger et al., 1981). However, the embryos themselves are still small enough for convenient histological processing.

Neural crest lesions. Eggs were opened with a hand drill fitted with a sanding disc. Under a sterile hood one or two drops of $1 \%(w / v)$ neural red in PBS were added to each egg to visualize the embryo and the condensed neural crest. Neural crest material was ablated by lightly touching the top of the spinal cord with an ophthalmological cautery unit (Concept, Inc., Clearwater, FL) fitted with a thin adaptor of stainless steel wire. Cauterization was done while the crest material was condensed on the dorsal surface of the cord. This siluation is found at the level of the two most caudal segmented somites and the next equivalent length of unsegmented somitic material (Tosney, 1978). To remove neural crest material that will comprise ganglia 15 to 17 (G15, G17), cauterization is done at stages 13 and 14 at the level of somites 20 to 23 (Yntema and Hammond, 1947). The eggs were then sealed with cellophane tape and reincubated until 9 days of incubation when the embryos were fixed.

During fixation the embryos were staged and examined for gross abnormalities including malformations of the wing. Cauterization procedures affected wing structure in some of the embryos (see also Shoobridge et al., 1983); to eliminate possible effects such structural abnormalities might have on the parameters examined, all embryos with wing deformities were discarded. To this effect, normal shoulderto-elbow and elbow-to-wing tip lengths at stage 35 were taken to be 5 $\mathrm{mm}$ and $9 \mathrm{~mm}$, respectively. Embryos showing any other gross deformities, with the exception of some slight axial twisting resulting from the cauterization procedures, were also discarded.

Control embryos were treated identically to experimentals except that cauterization was not performed.

Histological procedures. Embryos were fixed in Carnoy's solution and then processed by standard histological techniques. For all but one embryo (no. 900) Histosol (HS-100; National Diagnostics, Somerville, NJ) was used in place of xylene as the clearing agent because of the greatly enhanced clarity of histological detail it produces (Wishe et al., 1980). Embryos were embedded in Paraplast and sectioned at $7 \mu \mathrm{m}$ orthogonal to the axis of the spinal cord. Serial sections from all embryos except nos. 900 and 916 were stained by the Feulgen procedure (modified by Deitch et al., 1968) followed by fast green FCS counterstain. The sections from nos. 900 and 916 were stained with cresyl violet.

Identification of ganglia. For each embryo a two-dimensional scale reconstruction was made of the region encompassing G10 through $\mathrm{G} 17$ or G18, including the spinal nerves and brachial plexus. Ganglia were identified on the basis of their contribution to the brachial plexus (usually G13 to G16), the location of the lateral motor column, and the position of the wings. In controls, as well as many of the lesioned embryos, identification could also be made on the basis of ganglion size.

Ganglionic volume. The outlines of alternate sections through G11 to G17 of each embryo were projected onto paper and traced using a drawing tube attached to the microscope. The cross-sectional areas of these outlines were determined with a planimeter. Individual areas were summed, multiplied by 2 , corrected for the magnification, and multiplied by the section thickness to give the approximate volume of each ganglion.

Neuronal cell counts. Total numbers of nondegenerating neurons were estimated for G13 and G15 in all embryos. Neurons were identified by their large pale nuclei and pronounced nucleoli. Nuclei of nonneuronal cells are smaller, have smaller neucleoli, and are much more darkly staining. Counts were usually made in every sixth section through a ganglion and included all neuronal nuclei showing a nucleolus in that section. However, because of extremely small ganglion size resulting from the ablation procedures, counts in two of the ganglia were made in every second (left G15, no. 980) or third (right G15, no.
976) section. The mean number of neurons per section was determined and multiplied by the total number of sections to give an estimate of the total neuron number in each ganglion. Separate tabulations of lateroventral (LV) and mediodorsal (MD) neurons were also made because of their different developmental patterns in both control and experimental situations (Carr and Simpson, 1978a, b; Hamburger et al., 1981). No correction factors were applied to these counts because of the smaller nucleolar diameter $(\sim 1 \mu \mathrm{m})$ compared to the section thickness $(7 \mu \mathrm{m})$.

Cell degeneration. Total counts of degenerating cells were made in G11, G13, G14, and G15. Again, separate determinations were made for the $L V$ and MD regions. Cells counted as degenerating have been fully described previously (Carr and Simpson, 1978a). Briefly, they included pyknotic cells, vacuolated and fragmented cells, and debrisladen macrophages. It was assumed that at stage 35 most of these degenerating cells are neurons. It is not until later times that frankly degenerating non-neuronal cells appear (Hamburger et al., 1981).

Neuronal nuclear diameter. The average neuronal nuclear diameter was used as an index of neuronal cell size (Hughes and Tschumi, 1958; Hendry and Campbell, 1976). Determinations were carried out in G11, $\mathrm{G} 13$, and G15. The centermost section through a ganglion (or as close to it as possible) was examined under oil, and the outlines of all neuronal nuclei showing a nucleolus in that section were traced using a drawing tube. The longest and shortest diameters of each nucleus were then measured, and the average was determined. Data from the $\mathrm{LV}$ and $\mathrm{MD}$ regions of each ganglion were tabulated separately because of the well known differences in neuronal sizes of the two regions. A mean diameter was calculated for each region in each ganglion. From these values the overall means were determined.

\section{Results}

Gross morphology. The extent of injury inflicted by the cauterization procedures varied between embryos. In most embryos, ganglia were greatly reduced or missing along two axial segments at the site of lesion, while those immediately adjacent to the lesion appeared normal or nearly normal in size (Fig. 1).

Cord injury resulting from cauterization also varied between embryos. Efforts were made to keep such injury to a minimum. Two embryos (900 and 916) showed no obvious cord damage. The remaining embryos showed varying degrees of cord involvement ranging from slight reduction of the dorsal region to complete absence of the cord for, in some cases, up to one segment in length. In most embryos this cord damage was confined to levels caudal to G15 (Figs. 1 and 3). Histological evidence of muscle atrophy was not apparent in any embryos examined.

In normal stage 35 chick embryos the brachial plexus is comprised of spinal nerves from segments 13 through 16 . The contribution of spinal nerve 17 (SN17) to the plexus is variable. In the present experiments two of the four control embryos showed an SN17 contribution to the plexus. In previous studies such a contribution was never observed at this stage in normal embryos. In contrast, in the experimental embryos of the present study, SN17 consistently projected to the plexus.

Spinal nerve size depended on the axial level examined. Although direct quantitative measurements were not made, the fact that changes had occurred in the relative sizes of these nerves was quite apparent from gross histological observation as well as in the two-dimensional reconstructions. Spinal nerve enlargement was most pronounced in SN13 and was greatest when G13 was most hypertrophied. Conversely, spinal nerves were smallest where ganglia were most reduced.

Ganglionic volumes. The means \pm the standard deviations $(\overline{\mathbf{x}} \pm \mathrm{SD})$ of the volumes of G11 to G17 are given in Table I. Two points are immediately apparent. First, G15, G16, and G17 clearly show the direct effects of the cauterization procedures. Mean volumes are reduced by 30 to $40 \%$. For G16 this reduction is significant at the $0.05>p>0.025$ level of confidence (Student's $t$ test). For G15 and G17 the reductions are not statistically significant. Especially in the case of G15, 

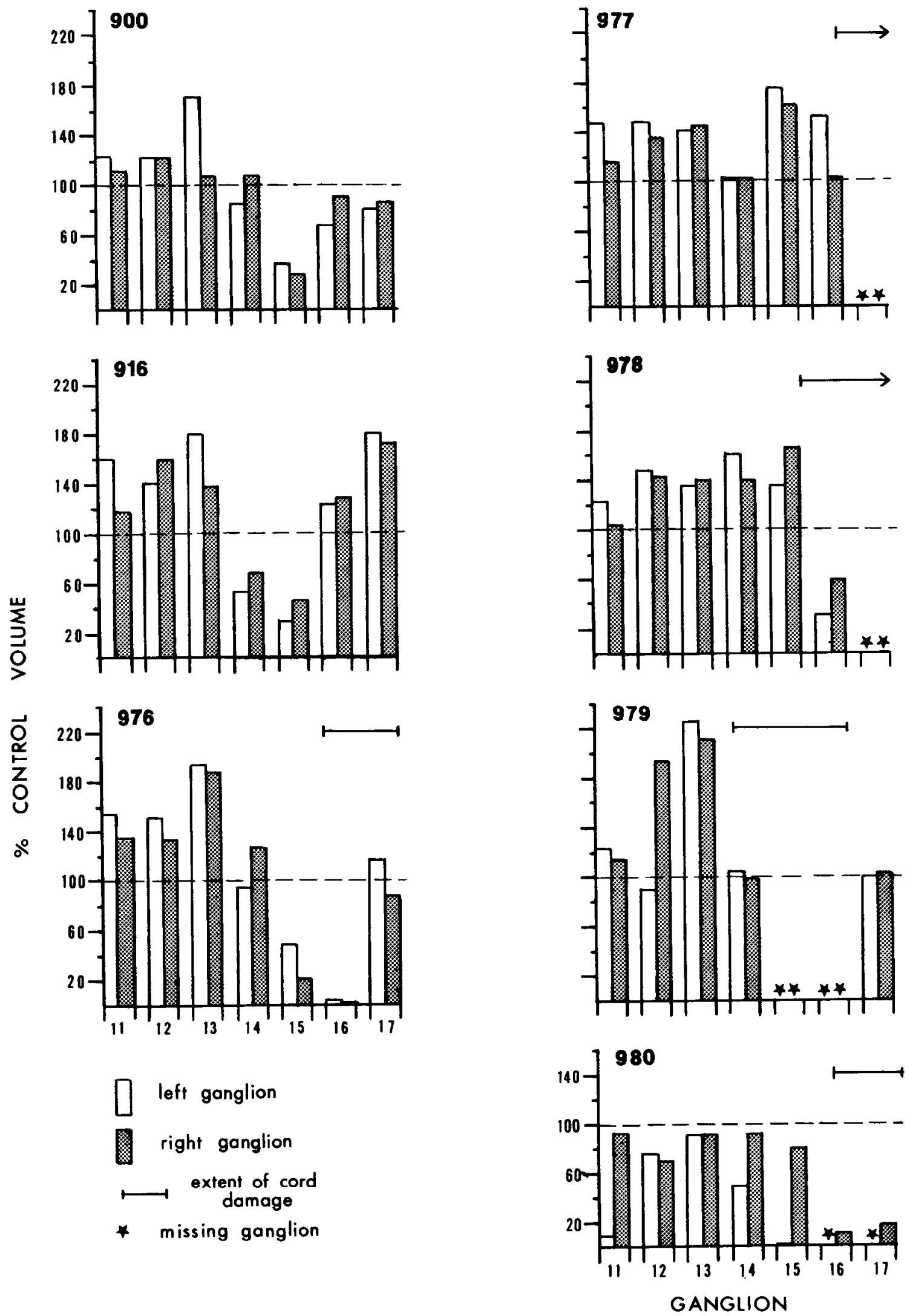

Figure 1. The volume of individual DRG in each experimental embryo relative to the mean volume of the corresponding ganglia in control embryos. Experimental embryos had been subjected to partial lesions of the brachial neural crest at stage 13 or 14 and allowed to develop to stage 35 . The extent of cord injury is indicated where appropriate. 
however, this lack of significance clearly reflects the large standard deviation due to between-animal differences in the exact placement and degree of the lesion. This variation is apparent in Figure 1. Moreover, G15 shows a significant reduction in neuronal cell numbers (see below).

The second salient point of Table I is that hypertrophies occur in ganglia well removed from the site of lesion. Ganglion 13 shows the largest response, an increase of $66 \%$ over the mean control value for the ganglion. This response is significant at the $0.05>p>0.025$ level of confidence. Cervical ganglia 11 and 12 also show the hypertrophies of 30 and $40 \%$, respectively. In G11 this hypertrophy is significant at $0.01>p>0.005$.

Among individual embryos G13 also shows the largest and most consistent response (Figs. 1 And 3). The degree of this response appears to reflect, in part, the location of the lesion relative to $\mathrm{G} 13$. The largest $\mathrm{G} 13$ responses occur when both G15 and G16, the largest brachial ganglia, are reduced (embryos 976 and 979). When the lesion is several segments away and other ganglia are able to respond to it (embryos 976 and 979). When the lesion is several segments away and other ganglia are able to respond to it (embryos 977 and 978), there is little effect on G13. More rostral placement of the lesion (no. 916) results in enlargement of G17.

The G13 response also reflects the magnitude of the lesion, as shown by Figure 2. In control embryos the volume of G13 appears to be directly proportional to the sum of the volumes of G14 through G17 (linear regression coefficient, $r=0.82$; significant at $0.05>p>0.025$ ). The size of $\mathrm{G} 13$ simply reflects that of the remaining ganglia innervating the wing and shoulder. In all probability these volumes are proportional to the size of the embryo. However, following neural crest ablation, the volume in G13 in each embryo is inversely proportional to the sum of the ganglionic material remaining in G14 through G17 ( $r=-0.75$, significant at $0.025>p>0.01)$. That is, G13 appears to be making compensatory volume adjustments in response to the reduced sizes of the brachial ganglia.

Only one embryo, no. 980, appears to show no responses to neural crest lesion (Fig. 1). However, in that embryo ganglia were smaller than normal, and, on the left side, cervical ganglia were fused for several segments. Ganglia on the right side, although small, were within the normal range of variation of control values ( $\sim 30 \%$ of the mean) and may indeed show a slight response to lesions at the level of G16 to G18. A similar situation occurs unilaterally in one control embryo. (Both sets of data were omitted from the statistical treatment of data throughout this paper).

Micrographs of hypertrophied and reduced ganglia are shown in Figure 3.

Neuronal cell counts. Neuronal cell counts (Table II) show that in G13 an increase in neuronal numbers of $24 \%$ occurs following neural crest lesions at the more caudal brachial levels. This hyperplasia is significant at the $0.05>p>0.025$ level of confidence. Similar hyperplasias occur in the LV and MD neuronal populations as well, although those changes are not statistically significant by themselves. In $\mathrm{G} 15$ a $45 \%$ reduction in neuronal numbers occurs. Reductions of 50 and $42 \%$, re-

TABLE I

Ganglionic volumes (mean $\pm S D \times 10^{6} \mu^{3}$ ) at stage 35 in control ganglia and following partial neural crest ablations at the level of G15 to G17

\begin{tabular}{lccccccc}
\hline \multicolumn{1}{c}{ Ganglion } & G11 & G12 & G13 & G14 & G15 & G16 \\
\hline Control & $4.6 \pm 0.1(5)^{a}$ & $4.5 \pm 1.4(5)$ & $5.0 \pm 1.4(6)$ & $10.2 \pm 2.7(7)$ & $13.2 \pm 3.4(7)$ & $12.2 \pm 2.9(7)$ & $7.0 \pm 1.6(7)$ \\
Experimental & $5.9 \pm 0.8(12)$ & $6.2 \pm 1.1(12)$ & $8.3 \pm 1.8(12)$ & $10.5 \pm 3.0(12)$ & $9.4 \pm 9.0(12)$ & $7.7 \pm 6.9(12)$ & $5.1 \pm 4.6(9)$ \\
$\begin{array}{l}\text { Percentage of control } \\
\text { value }\end{array}$ & 128.3 & 137.8 & 166.0 & 102.9 & 71.2 & 63.1 \\
Level of confidence & $0.01>p>0.005$ & $\mathrm{NS}^{b}$ & $p<0.005$ & NS & NS & $0.05>p>0.025$ & NS \\
\hline
\end{tabular}

${ }^{a}$ Numbers in parentheses represent the number of ganglia examined.

${ }^{b}$ NS, not significant.

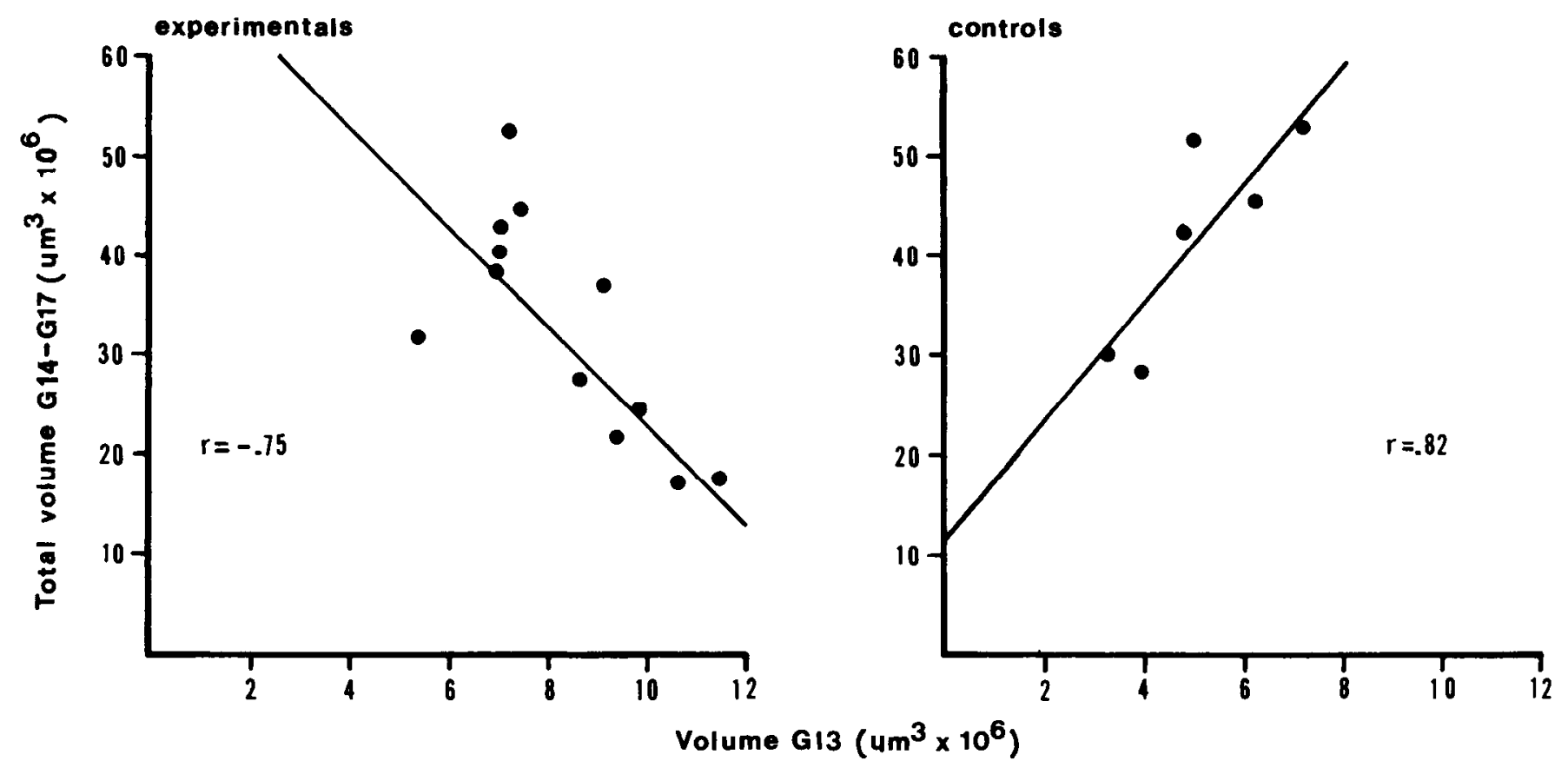

Figure 2. The volume of G13 versus the sum of the volumes of G14 through G17 in individual embryos. Experimental embryos had been subjected to partial neural crest ablations at the level of G15 to G17. $r=$ linear correlation coefficient. 

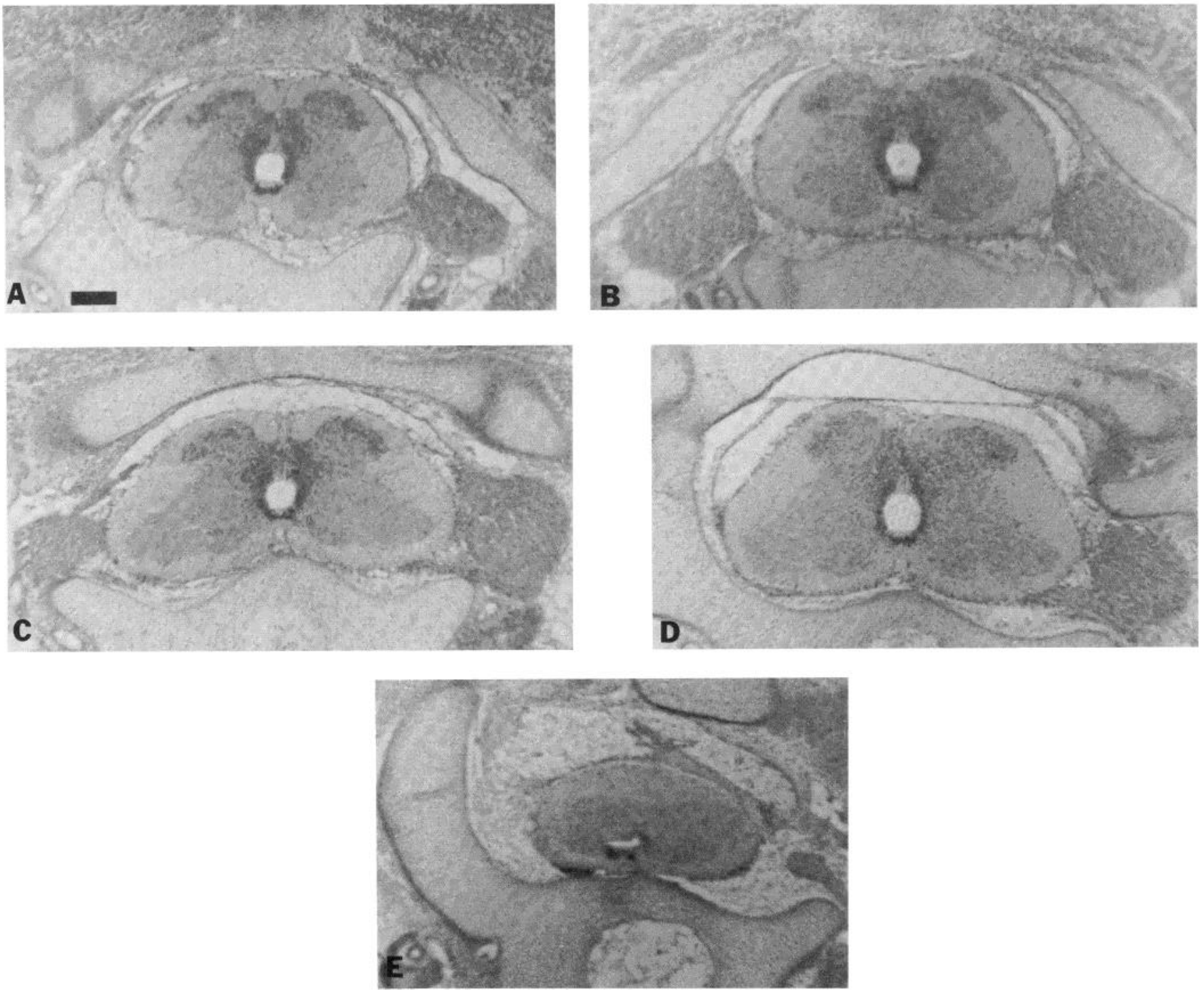

Figure 3. Cross-sections through the spinal cord and DRG in stage 35 control $(A$ and $C)$ and experimental $(B, D$, and $E)$ embryos. $A$ to $D$, The middle sections of the left ganglion (on viewer's right) in G13 ( $A$ and $B$ ) and G15 $(C$ and $D)$. Control ganglia were chosen because their volumes were close to the mean control volumes. Ganglionic hypertrophy of G13 and reduced size of G15 in the experimental embryos are noticeable. (The apparent absence $(A$ and $D)$ or smaller size $(B$ and $C)$ of the right versus left ganglia is due to the plane of section of these embryos.) $B$ and $D$ also illustrate the grossly normal appearance of the spinal cord at these levels in these particular experimental embryos. The normality of the cord at the level of G15 $(D)$ despite reduced size of G15 is especially striking in comparison to the obviously damaged cord (and very much reduced left ganglion) at the level of G16 (E) in the same embryo. $A$ and $C$, embryo $944 ; B$, embryo $979 ; D$ and $E$, embryo 976 . Calibration bar $=100 \mu \mathrm{m}$.

spectively, occur in the LV and MD populations. All represent significant changes. In both sets of ganglia, linear regression analysis (data not shown) shows a direct relationship between ganglionic volume and neuron number as, indeed, one would expect.

Cell degeneration. The mean numbers of degenerating cells in each ganglion are given in Table III. Ganglion 15 was included in this study because it is normally the largest brachial ganglion and because in most of the experimental embryos it was located within the lesion site. Ganglion 14 was included because it shows little or no ganglionic hypertrophy despite its close proximity to the site of lesion. Ganglion 13 was included because hypertrophic responses to the neural crest lesions occur there more consistently than in any other ganglion despite its remoteness from the lesion site. It also shows the significant hyperplasia described above. Moreover, previous studies have shown the G13 proliferative and degenerative patterns to be those of cervical rather than brachial ganglia. This is true in both normal embryos and following wing bud amputation (Carr and Simpson, 1978a, b). Finally, G11 was included in this study because, although well removed from the lesion site, it, nonetheless, also shows slight hypertrophic responses.

Results show that no changes occurred in the number of degenerating cells in either G11 or G13. This was true of whole ganglia and of the LV and MD regions as well. In G14, 25 to $30 \%$ decreases in numbers of degenerating cells were observed. None of these was statistically significant. Gangion 15 , in contrast, showed significant reductions, of 40 to $50 \%$, in all three sets of data. However, with the exception of a single ganglion (left G15, no. 980), in no case was a total absence of degenerating cells observed.

However, G15 differs from the other ganglia in that it was directly affected by the ablation procedures and consequently shows a wide range of ganglionic volumes. As a result it is 
TABLE II

Mean neuronal numbers $\pm S D$ in entire ganglia and in $L V$ and $M D$ regions at stage 35 in control ganglia and following partial neural crest ablations

\begin{tabular}{|c|c|c|c|}
\hline Ganglion & Entire ganglion & Lateroventral & Mediodorsal \\
\hline \multicolumn{4}{|l|}{ G13 } \\
\hline Control $(6)^{a}$ & $6,490 \pm 1,872$ & $2,236 \pm 586$ & $4,253 \pm 1,505$ \\
\hline Experimental (12) & $8,040 \pm 1,114$ & $2,719 \pm 755$ & $5,319 \pm 902$ \\
\hline $\begin{array}{l}\text { Percentage of control } \\
\text { value }\end{array}$ & 123.9 & 121.6 & 125.1 \\
\hline Level of confidence & $0.05>p>0.25$ & $\mathrm{NS}^{b}$ & NS \\
\hline \multicolumn{4}{|l|}{$\mathrm{G} 15$} \\
\hline Control (7) & $13,034 \pm 1,965$ & $4,140 \pm 461$ & $8,894 \pm 1,749$ \\
\hline Experimental (12) & $7,217 \pm 6,498$ & $2,075 \pm 1,885$ & $5,142 \pm 4,647$ \\
\hline $\begin{array}{l}\text { Percentage of control } \\
\text { value }\end{array}$ & 55.4 & 50.1 & 57.8 \\
\hline Level of confidence & $0.01>p>0.005$ & $0.005>p$ & $0.25>p>0.0$ \\
\hline
\end{tabular}

TABLE III

Mean numer of degenerating cells $\pm S D$ per ganglion and in $L V$ and $M D$ regions at stage 35 in control ganglia and following partial neural crest ablations

\begin{tabular}{lccc}
\multicolumn{1}{c}{ Ganglion } & Entire Ganglion & Lateroventral & Mediodorsal \\
\hline $\mathrm{G} 11$ & & & \\
Conrol (5) & & & \\
Experimental (8) & $84.2 \pm 20.5$ & $21.6 \pm 5.2$ & $68.4 \pm 21.3$ \\
Level of confidence & $\mathrm{NS}^{b}$ & $\mathrm{NS}$ & $\mathrm{NS}$
\end{tabular}

G13

$\begin{array}{lccc}\text { Control (6) } & 120.8 \pm 61.3 & 42.0 \pm 16.8 & 71.6 \pm 47.4 \\ \text { Experimental (8) } & 132.8 \pm 59.3 & 37.1 \pm 21.3 & 87.0 \pm 34.8 \\ \text { Level of confidence } & \text { NS } & \text { NS } & \text { NS }\end{array}$

G14

$\begin{array}{lccc}\text { Control (7) } & 160.9 \pm 75.4 & 44.9 \pm 19.0 & 112.0 \pm 57.8 \\ \text { Experimental (8) } & 115.3 \pm 69.4 & 29.2 \pm 15.4 & 84.0 \pm 56.2 \\ \text { Level of confidence } & \text { NS } & \text { NS } & \text { NS }\end{array}$

G15

\begin{tabular}{lccc} 
Control (7) & $185.1 \pm 38.3$ & $24.0 \pm 7.8$ & $159.7 \pm 36.0$ \\
Experimental (6) & $100.0 \pm 49.4$ & $15.0 \pm 3.9$ & $84.0 \pm 46.3$ \\
Level of confidence & $p<0.005$ & $0.025>p>0.01$ & $p<0.005$ \\
\hline
\end{tabular}

${ }^{a}$ Numbers in parentheses are numbers of ganglia counted.

${ }^{b}$ NS, not significant.

possible that the total number of degenerating cells G15 contains may not accurately reflect its true level of degenerative activity. Ganglia directly affected by the ablation procedures, such as G15, could contain reduced numbers of degenerating cells simply because they are smaller rather than because their neurons have been differentially spared from cell death. In such a case the proportion of total neurons that are degenerating would not change. On the other hand, if the constituent neurons of a ganglion were responding to reduced competition (e.g., as a result of the lesion) and were doing so by undergoing less cell death, then the proportion as well as the number of neurons degenerating within the ganglion should be reduced.

For this reason the proportions of neurons degenerating in G13 and G15 were also determined. The total number of neurons in each individual ganglion was obtained by adding the number of healthy neurons (above) and the number of degenerating cells. The percentage of total neurons that was degenerating was determined, and the individual values were averaged. By expressing the percentage of neurons degenerating in terms of total rather than of only healthy neurons, we obtained a figure comparable to the degeneration index determined by the author in previous studies (Carr and Simpson, 1978a, b).

Results are given in Table IV. Values obtained from control embryos do not differ from those found previously (Carr, 1976). In experimental embryos, while no significant changes occur in G13 (total, LV, or MD) or the LV region of G15, significant reductions do occur in G15 in both total ganglion and $\mathrm{MD}$ degeneration indices. These reductions are $33 \%$ and $43 \%$, respectively.

Average neuronal nuclear diameter. Neural crest ablations also have pronounced effects on neuronal sizes (Table V, Fig. 4). In G13 and G15 hypertrophies of 0.7 to $1 \mu \mathrm{m}$ occur, which are quite significant. These responses represent 11 to $16 \%$ enlargements of the average nuclear diameter of the $\mathrm{LV}$ and MD neuronal populations of both ganglia. In G11 the hypertrophies are smaller, of less than $0.5 \mu \mathrm{m}$. Only the response of the $L V$ region is significant, and that is at a relatively low level of confidence.

These observations are clearly reflected in the percentage distributions of average nuclear diameters shown in Figure 5. Striking shifts can be seen in the neurons of both G15 and G13, but not those of G11.

\section{Discussion}

The results reported above demonstrate that significant hypertrophies occur in DRG material that remains following partial ablations of the brachial neural crest. Ganglionic hypertrophy has been reported for chick DRG following addition of supernumerary limbs at lumbosacral and thoracic levels (Hamburger, 1939a, b; Hamburger and Levi-Montalcini, 1949; Hollyday and Hamburger, 1976) and following hemisection of the brachial cord and removal of the associated neural crest (Castro, 1963). The numerous other cases of hypertrophy of neural centers have been reviewed extensively (Hughes and Carr, 1978; Oppenheim, 1981; Hamburger and Oppenheim, 1982).

It seems noteworthy that the largest hypertrophies occurred not in ganglia immediately adjacent to the lesioned region, but rather in more remote ganglia, as far rostral as G11. Similar observations were not reported by Castro (1963). Such hypertrophies as reported here indicate that these more remote ganglia are making compensatory responses to the reduction of ganglionic material at the level of the lesions. The inverse and statistically significant relationship between the volume of G13 and the summed volumes of G14 through G17 in experimental embryos (Fig. 2) supports this conclusion.

Why ganglia closer to the the lesion site are of normal size is not clear. It is possible that these apparently normal ganglia have, in fact, been slightly affected by the neural crest ablations but that they have been able to compensate for such effects.

\section{TABLE IV}

Degeneration indices $\pm S D$ in entire ganglia and in $L V$ and $M D$ regions at stage 35 in control ganglia and following partial neural crest ablation

\begin{tabular}{lccc}
\hline \multicolumn{1}{c}{ Ganglion } & Entire Ganglion & Lateroventral & Mediodorsal \\
\hline G13 & & & \\
Control (6) & & & \\
Experimental (8) & $1.8 \pm 0.5$ & $1.9 \pm 0.9$ & $1.7 \pm 0.8$ \\
Level of confidence & $1.6 \pm 0.6$ & $1.4 \pm 1.1$ & $1.7 \pm 0.7$ \\
G15 & $\mathrm{NS}^{b}$ & $\mathrm{NS}$ & $\mathrm{NS}$ \\
Control (7) & $1.4 \pm 0.2$ & $0.6 \pm 0.2$ & $1.8 \pm 0.3$ \\
Experimental (6) & $0.9 \pm 0.2$ & $0.7 \pm 0.5$ & $1.0 \pm 0.2$ \\
Level of confidence & $p<0.005$ & $\mathrm{NS}$ & $p<0.005$ \\
\hline
\end{tabular}

${ }^{a}$ Numbers in parentheses are numbers of ganglia counted.

${ }^{b}$ NS, not significant. 
TABLE V

Average neuronal nuclear diameter (micrometers) $\pm S D$ in stage 35 embryos following partial neural crest ablation Each value is based on data from the centermost section of 5 to 12 ganglia.

\begin{tabular}{cccccc}
\hline Region & Ganglion & Controls & Experimentals & $\frac{E}{C} \times 100 \%$ & $\begin{array}{c}\text { Level of } \\
\text { confidence }\end{array}$ \\
\hline Lateroventral & G11 & $7.2 \pm 0.3(409)^{a}$ & $7.6 \pm 0.2(1214)$ & 105.6 & $0.05>p>0.025$ \\
& G13 & $6.8 \pm 0.4(514)$ & $7.6 \pm 0.5(1379)$ & 111.8 & $0.01>p>0.005$ \\
Mediodorsal & G15 & $7.3 \pm 0.6(778)$ & $8.1 \pm 0.4(859)$ & 111.0 & $p<0.005$ \\
& G11 & $6.1 \pm 0.3(899)$ & $6.4 \pm 0.2(2110)$ & 104.9 & NS \\
& G13 & $5.9 \pm 0.6(792)$ & $6.6 \pm 0.4(2213)$ & 111.9 & $0.025>p>0.01$ \\
& G15 & $6.2 \pm 0.6(1465)$ & $7.2 \pm 0.4(1420)$ & 116.1 & $p<0.0005$ \\
\hline
\end{tabular}

${ }^{a}$ Numbers in parentheses represent the numbers of nuclei measured.
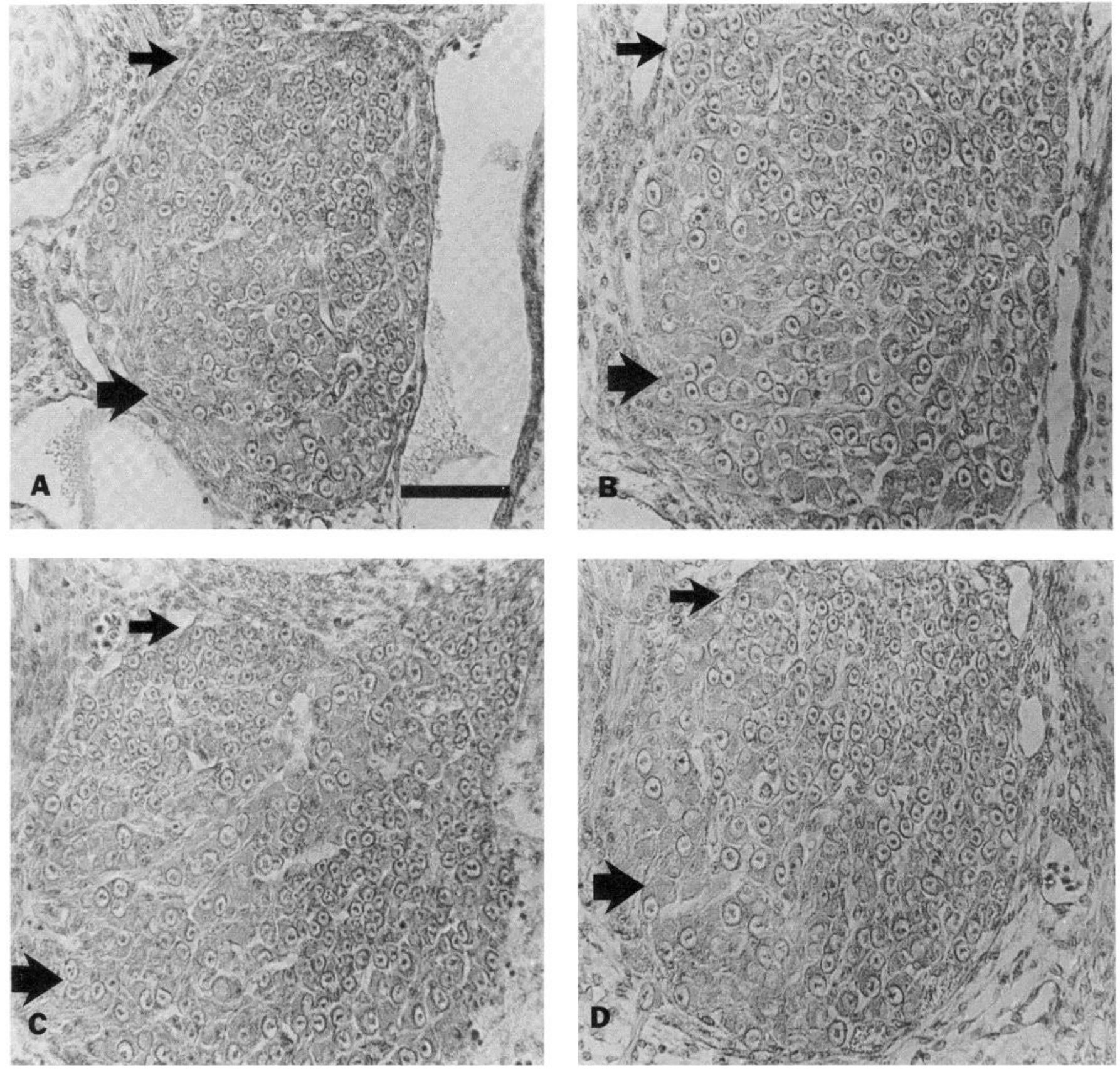

Figure 4. Enlargements of the left ganglia shown in Figure 3, $A$ to $D$ demonstrating the neuronal hypertrophy in experimental G13 ( $B)$ and $\mathrm{G} 15(D)$ compared to control ganglia $(A$ and $C)$. Calibration line $=50 \mu \mathrm{m}$. Large arrow, LV region; small arrow, MD region. 

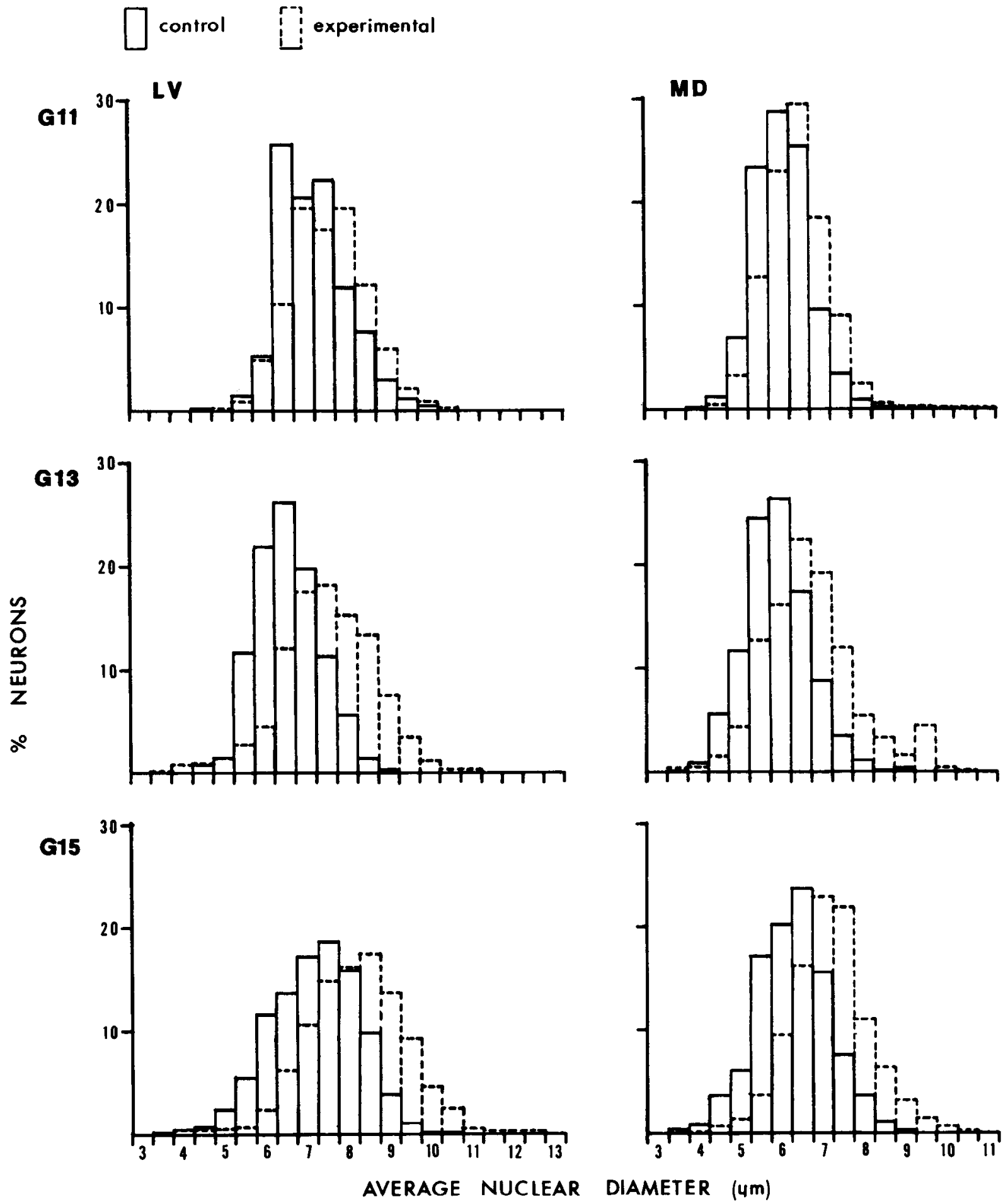

Figure 5. The percentage distribution of the average nuclear diameters of neurons in stage 35 control DRG and in DRG of embryos subjected to partial neural crest lesions. Data are presented separately for neurons in the LV and MD regions of the ganglia.

Indeed G14, which shows no change in ganglionic volume, does show $25 \%$ to $35 \%$ reductions in numbers of degenerating cells, although none of these reductions is statistically significant. However, if G14 is compensating for small direct injuries, it is curious that this ganglion was not able to respond sufficiently to the absence or reduction of its neighbors to show any significant hypertrophy.

The ganglionic hypertrophies observed clearly reflect compensatory cellular changes occurring within these ganglia.
These changes include neuronal hyperplasia and hypertrophy. As mentioned, the volume of individual ganglia in both control and experimental embryos correlates closely with the number of neurons they contain. Ganglion 13 shows a significant increase in the mean number of neurons in experimental embryos over control values $(\sim 8000$ versus 6500$)$. Increases of similar magnitudes occurred within both the LV and MD populations, although by themselves these were not significant. Such a hyperplasia coupled with the observed $12 \%$ neuronal nuclear 
hypertrophy would account for the degree of ganglionic hypertrophy observed.

It is presumed that such hyperplasias result from the sparing of neurons that would have otherwise died during the normal course of development in these ganglia (Hamburger and LeviMontalcini, 1949; Carr and Simpson, 1978a; Hamburger et al., 1981). According to the well known competition hypothesis (Hamburger and Levi-Montalcini, 1949; Cowan, 1973), this sparing is due to decreased competition between sensory fibers within the DRG peripheral targets for limited amounts of trophic substances such as nerve growth factor (NGF) (Gorin and Johnson,1980; Johnson et al., 1980; Hamburger et al., 1981; Oppenheim et al., 1982) or other agents (Barde et al., 1978, 1980). By reducing the amount of neural crest material available for formation of the DRG, the cauterization procedures would have reduced the numbers of neurons competing for the trophic agent(s). Hence, cell death would be reduced in the remaining ganglionic material.

However, despite its $24 \%$ neuronal hyperplasia, G13 showed no change in either the absolute number of degenerating cells or in the degeneration indices in either the total ganglion or in the LV or MD regions when examined at the stage 35 . However, it must be kept in mind, that the cell number (as well as ganglionic and neuronal volumes) reflects the accumulation of events operating over a period of time. Thus, it is quite likely that degenerative responses had already occurred during the 5 days between the onset of degenerative activity at stage 24 (Carr and Simpson, 1978a, b; Hamburger et al., 1981) and the time of examination at stage 35 . The same argument could also be made for G11, which similarly shows ganglionic hypertrophy but no degenerative responses at stage 35 . If G14 proves to have been slightly injured and to have responded to such injury as discussed above, the same argument would apply to it as well. Experiments are planned to examine the earlier stages. It would be surprising if reductions in degenerative activity were not found.

In contrast, G15 shows a striking decrease in the degenerative activity of the ganglion as a whole, as well as of the MD region. This is true of both the degeneration indices and total numbers of degenerating cells. The large decrease in degenerative activity in the $\mathrm{MD}$ region compared to that in the $\mathrm{LV}$ region is in accord with the different responses seen in the two regions at 35 by Hamburger and co-workers (1981) following exposure of chick embryos to NGF.

This striking reduction in degenerative activity in G15 at stage 35 is strong evidence for the sparing of neurons due to reduction of the number of competing neurons. Circumstantial evidence for such reduced cell death has been reported in the ciliary ganglion of the chick following selective axotomy of nerve branches to the iris and ciliary muscles of the eye (Pilar et al., 1980) and in the lateral motor column of Xenopus following transection of the spinal nerve to a neighboring segment (Olek and Edwards, 1977). Although in neither case were actual counts of degenerating cells made, the conclusions were based on neuronal counts made before and after the period of normally occurring neuronal cell death. In contrast, no significant changes in degenerative activity were found in hypertrophied and hyperplastic DRG of Rana pipiens following uniteral removal of a nearby ganglion (Bibb, 1978; Davis and Constantine-Paton, 1983a).

Our results also demonstrate another widely observed phenomenon: an apparent inability of the procedures to spare all neurons from cell death despite the large reduction in competition at the periphery (see Oppenheim, 1981). Given the large decreases in neuron number and ganglion volume in G15 due to the neural crest lesions, total loss of degenerative activity might have been expected. This was not observed. Moreover, the one exceptional ganglion was so small $\left(0.3 \times 10^{6} \mu \mathrm{m}^{3}\right)$ that the absence of degenerating cells from it could well have been more of a sampling size problem than a real phenomenon.

Such observations are usually attributed to a neuron's inability to successfully innervate inappropriate targets, to its having limited access to appropriate targets (Hollyday and Hamburger, 1976; Oppenheim, 1981) or to a nonuniform innervation density at the target (Pilar et al., 1980). These are all situations that might occur during innervation of supernumerary limbs. However, in the case of a greatly reduced number of neurons confronting their original, intact targets, as occurs with neural crest ablations, the possibility also arises that some innervating neuronal populations, or subpopulations, may be somewhat limited in their capacity for expansion of their peripheral innervation. The apparent maximal level of cell sparing found in the present experiments in partially ablated G15 would support such a possibility. The fact that G14 also showed no hypertrophy and no significant decrease in degenerative activity despite the greatly reduced size of G15 in several embryos may provide further support. Direct evidence for limited neuronal expansion capabilities comes from Scott's observations (1984) of differential expansion of various dermatomes in both horseradish peroxidase (HRP) and electrophysiological studies following neural crest lesions at the lumbosacral level.

It has also been suggested that the persistent degenerating cells might be cells that die before contacting their peripheral targets. Such cells would not then be spared by reducing neuronal competition. Our own observation of cells that degenerate within only a few hours of undergoing DNA synthesis (Carr and Simpson, 1982) might seem to support this possibility. However, the rapidly degenerating cells we observed were only seeen earlier in development, at $4 \frac{1 / 2}{2}$ and $5 \frac{1}{2}$ days of incubation. 'They were not observed as late as $81 / 2$ days. Moreover, the rapidly degenerating cells did not appear to represent a programmed cell death. Rather, their death appeared to be an indirect response to alterations at the periphery. Finally, we have also shown that DRG neuronal production ceases by $7 \frac{1}{2}$ days of incubation (Carr and Simpson, 1978a). It seems likely that if substantial numbers of neurons were degenerating without having made peripheral contact as late as stage $35(81 / 2$ to 9 days), they would have had a noticeably immature appearance prior to the onset of their degeneration. Such a population of immature neurons was not observed. Rather, neurons in stage 35 DRG show a fairly uniform degree of maturation consistent with their location in the LV or MD regions. Thus, it would seem that few, if any, of the degenerating cells observed in the much reduced DRG at stage 35 are likely to represent a neuronal population that has failed to establish peripheral contact.

A final possibility for the persistent degeneration is that it is the result of cord damage that occurred in several embryos. This point is discussed below along with other possible effects of cord damage.

The other cellular response to partial neural crest ablation that was measured was the average neuronal nuclear diameter. Like the ganglionic volumes, this measure of neuronal size (Hughes and Tschumi, 1958; Hendry and Campbell, 1976) indicates that significant neuronal hypertrophy occurred at least as far rostrally as G11. However, responses were most pronounced at levels closer to or at those of the lesions, in G13 and G15.

Neuronal hypertrophy has been reported in other systems following enlargement of the peripheral field (e.g., Cavanaugh, 1951; Hughes, 1962; Pannese, 1963; Pollack, 1969; Hollyday and Mendell, 1976). In adult animals such neuronal hypertrophies have been correlated with increased functional activity in response to increased demands (Pannese, 1963). In embryonic material enhanced neuronal maturation is likely to be involved as well. However, neuronal hypertrophy was found not to accompany reduction of neuronal cell death in either the 
chick ciliary ganglion following selective axotomy (Pilar et al., 1980 ) or the chick lateral motor column following neuromuscular blockade (see Oppenheim, 1981) despite enhanced axonal maturation in both systems.

One parameter not examined in the present study was proliferative activity. As stated above, the only proliferative response observed following early wing bud amputation was a reduced glial proliferation (Carr and Simpson, 1978b). Following partial neural crest ablations, one would similarly expect to see increased glial proliferative activity in the remaining ganglia in response to reduced neuronal degeneration and to neuronal hypertrophy (Pannese, 1964). However, although highly unlikely, the possibility of a neuronal proliferative response cannot be completely eliminated. First, proliferation of neuronal precursors and the establishment of peripheral connections occur simultaneously in the chick DRG. Second, Bibb (1977) has found an increase in the number of mitotic figures in $R$. pipiens DRG following ganglionectomy, although the identity of the proliferating cells was not determined. (However, others have been unable to demonstrate increased autoradiographic labeling with tritiated thymidine in similar experiments in this species (Davis and Constantine-Paton, 1983a).) Third, reduced degeneration indices were not observed in G13 in the present experiments despite a significant increase in neuronal number. As discussed above, however, degenerative responses may have occurred prior to stage 35 . Therefore, it is important that the possibility of increased neuronal proliferation following partial neural crest ablations be examined.

One factor that is difficult to assess in these experiments is the possible effect that spinal cord damage may have had. Whatever these effects may be, it is important to note that the ganglionic and neuronal hypertrophies, as well as the neuronal hyperplasia (G13) and reduced degenerative activity (G15) that were observed, are opposite from the regressive changes one might expect if cord damage were having any major effect at this stage. Nevertheless, many of the experimental embryos displayed variable amounts of cord damage as a result of the cauterization procedures. In most of the embryos the cord damage occurred caudal to G15 and, thus, caudal to the levels at which neuronal cellular responses were examined. At levels where damage does occur, the DRG are themselves already much reduced or missing (Fig. 1). It is conceivable that cord damage might account for the persistence of degenerative activity in these reduced ganglia. At other levels any effect on the DRG would depend on how far rostrally or caudally the central processes of those ganglia projected. Hulsebosch and Coggeshall (1983) have recently described sprouting of unmyelinated dorsal root axons in rats following transection of neighboring dorsal roots. This sprouting appears to be mediated through interactions within the spinal cord and is greater following transection of caudal than of rostral roots. Cord damage might also have an indirect effect on the DRG, due to muscle atrophy resulting from loss of the trophic functions of motor neurons. As noted under "Results," however, no evidence of muscle atrophy was observed in the present experiments. It is possible that it would appear at a later stage.

One of the most interesting points of the observations reported above is the indirect evidence they provide that alterations may have occurred in DRG peripheral projections in the experimental projections in the experimental embryos. First, some of the largest hypertrophies and cellular changes occurred in ganglia not immediately adjacent to reduced or missing ganglia but in more remote ones. Second, in most experimental embryos G13 and/or G17 showed evidence of increased brachial involvement. Ganglionic hypertrophies were found most consistently in G13 along with corresponding enlargement of SN13 (also see Castro, 1963). Accompanying these hypertrophies were significant hyperplasias and increased neuronal size.
Moreover, G17 contributed to the brachial plexus in every experimental embryo, a situation not seen in normal stage 35 embryos. Ganglion 17 also showed a large hypertrophy in one case where neural crest lesion was rostrally placed. Third, ganglionic responses occurred even in more rostrally located, cervical ganglia. Although these ganglia, G11 and G12, were never observed to contribute to the brachial plexus in any experimental embryos, the changes exhibited by them could reflect caudal shifts in their innervation patterns to compensate for increased G13 involvement in brachial innervations. Obviously, electrophysiological and HRP tracing studies are required to confirm these suggestions of altered peripheral projections at the brachial level. Such studies have recently been carried out at the lumbosacral level, however, by Scott (1984). She has found expansion of dermatomes within the hindlimb as well as shifts in the distribution of DRG axons within their appropriate pathways following deletions of lumbosacral neural crest. Moreover, Davis and Constantine-Paton (1983b) have found that the expansion of peripheral projections of lumbosacral DRG they observe following ganglionectomy in $R$. pipiens generally correlated with the degree of neuronal hyperplasia within the ganglia.

Finally, some attention should be focused on differences between the LV and MD regions of the DRG. The parallel between their degenerative responses in $\mathrm{G} 15$ in the present study and those following NGF administration (Hamburger et al., 1981) has already been noted. It is also interesting that the relative neuronal numbers in these two regions appear to remain constant, at least under the conditions examined here (Table II). The $L V$ region encompasses approximately 30 to $35 \%$ of the total neuronal populations in both G13 and G15 and in both experimental and control embryos. The MD region encompasses the rest. These two regions have yet to be fully defined either physiologically or pharmacologically. Nevertheless, the maintenance of these relative cell numbers suggests that this ratio is of importance. It will be of considerable interest to determine how this ratio is established and how it is maintained despite the (presumably) random removal of neural crest precursor material in the experimental embryos.

\section{References}

Barde, Y. A., R. M. Lindsay, D. Monard, and H. Thoenen (1978) New factor released by cultured glioma cells supporting survival and growth of sensory neurons. Nature 274: 818 .

Barde, Y. A., E. Edgar, and H. Thoenen (1980) Sensory neurons in culture: Changing requirements for survival factors during embryonic development. Proc. Natl. Acad. Sci. U. S. A. 77: 1199-1203.

Berg, D. K. (1982) Cell death in neuronal development, In Neuronal Development, N. C. Spitzer, ed., pp. 297-331, Plenum Press, New York.

Bibb, H. D. (1977) The production of ganglionic hypertrophy in Rana pipiens larvac. J. Exp. Zool. 200: 265270.

Bibb, H. D. (1978) Neuronal death in the development of normal and hyperplastic ganglia. J. Exp. Zool. 206: 65-72.

Carr, V. McM. (1976) Instrinsic and peripheral factors in the early development of the chick dorsal root ganglia. Ph.D. thesis, Northwestern University, Evanston, IL.

Carr, V. McM., and S. B. Simpson, Jr. (1978a) Proliferative and degenerative events in the early development of chick dorsal root ganglia. I. Normal development. J. Comp Neurol. 182: 727-740.

Carr, V. McM., and S. B. Simpson, Jr. (1978b) Proliferative and degenerative events in the early development of chick dorsal root ganglia. II. Responses to altered peripheral fields. J. Comp. Neurol. 182: $741-756$.

Carr, V. McM., and S. B. Simpson, Jr. (1982) Rapid appearance of labeled degenerating cells in the dorsal root ganglia after exposure of chick embryos to tritiated thymidine. Dev. Brain Res. 2: 157-162.

Castro, G. D. (1963) Effects of reduction of nerve centers on the development of residual ganglia and on nerve patterns in the wing of the chick embryo. J. Exp. Zool. 152: 279-293. 
Cavanaugh, M. W. (1951) Quantitative effects of the peripheral innervation area on nerves and spinal ganglion cells. J. Comp. Neurol. 94 187-219.

Cowan, M. W. (1973) Neuronal death as a regulative mechanism in the control of cell number in the nervous system. In Development and Ageing of the Nervous System, M. Rockstein, ed., pp. 19-41, Academic Press, Inc., New York.

Davis, M. R., and M. Constantine-Paton (1983a) Hyperplasia in the spinal sensory system of the frog. I. Plasticity in the most caudal dorsal root ganglion. J. Comp. Neurol. 221: 444-452.

Davis, M. R., and M. Constantine-Paton (1983b) Hyperplasia in the spinal sensory system of the frog. II. Central and peripheral connectivity patterns. J Comp. Neurol. 221: 453-465.

Deitch, A. D., D. Wagner, and R. M. Richert (1968) Conditions influencing the density of the Feulgen reaction. J. Histochem. Cytochem. 16: $371-379$.

Gorin, P. D., and E. M. Johnson, Jr. (1980) Effects of exposure to nerve growth factor antibodies on the developing nervous system of the rat: an experimental autoimmune approach. Dev. Biol. 80: 313323.

Hamburger, V. (1939a) The development and innervation of transplanted limb primordia in chick embryos. J. Exp. Zool. 80: 347-389.

Hamburger, V. (1939b) Motor and sensory hyperplasia following limbbud transplantations in chick embryos. Physiol. Zool. 12: 268-284.

Hamburger, V., and H. L. Hamilton (1951) A series of normal stages in the development of the chick embryo. J. Morphol. 88: 49-92.

Hamburger, V., and R. Levi-Montalcini (1949) Proliferation, differentiation and degeneration in the spinal ganglia of the chick embryo under normal and experimental conditions. J. Exp. Zool. 111:457502.

Hamburger, V., and R. W. Oppenheim (1982) Naturally occurring neuronal death in vertebrates. Neurosci. Comment. 1: 39-55.

Hamburger, V., J. K. Brunso-Bechtold, and J. W. Yip (1981) Neuronal death in the spinal ganglia of the chick embryo and its reduction by nerve growth factor. J. Neurosci. 1: 60-71.

Hendry, I. A., and J. Campbell (1976) Morphometric analysis of rat superior cervical ganglion after axotomy and nerve growth factor treatment. J. Neurocytol. 5: 351-360.

Hollyday, M., and V. Hamburger (1976) Reduction of the naturally occurring motor neuron loss by enlargement of the periphery. $\mathrm{J}$. Comp. Neurol. 170: 311-320.

Hollyday, M., and L. Mendell (1976) Analysis of moving supernumerary limbs of Xenopus laevis. Fxp. Neurol. 51: 316-329.

Hughes, A. (1962) An experimental study on the relationships between limb and spinal cord in the embryo of Eleutherodactylus martinicencis. J. Embryol. Exp. Morphol. 10: 575-601.

Hughes, A., and V. McM. Carr (1978) The interaction of periphery and center in the development of dorsal root ganglia. In Handbook of Sensory Physiology, Vol. 9, M. Jacobson, ed., pp. 85-114, SpringerVerlag, Berlin.

Hughes, A., and P. A. Tschumi (1958) The factors controlling the development of the dorsal root ganglia and ventral horn in Xenopus laevis (Daud.). J. Anat: (Lond.) 92: 498-527.

Hulsebosch, C. E., and R. E. Coggeshall (1983) Age related sprouting of dorsal root axons after sensory denervation. Brain Res. 288: 7783 .

Johnson, E. M., Jr., P. D. Gorin, L. E. Brandeis, and J. Pearson (1980) Dorsal root ganglion neurons are destroyed by exposure in utero to maternal antibody to nerve growth factor. Science 210: 916-918.

Levi-Montalcini, R., and G. Levi (1943) Recherches quantitatives sur la marche du processus de diffèrentiation des neurones dans les ganglions spinaux de l'embryon de poulet. Arch. Biol. 54: 198-206.

Morris, D. (1978) Development of functional motor innervation in supernumerary hind limbs of the chick embryo. J. Neurophysiol. 41 : $1450-1465$.

Olek, A. J., and C. Edwards (1977) The effect of spinal nerve section on motor neuron loss during development in Xenopus. Soc. Neurosci. Abstr. 3: 115.

Oppenheim, R. W. (1981) Neuronal cell death and some related regressive phenomena during neurogenesis: A selective historical review and progress report. In Studies in Developmental Neurobiology. Essays in Honor of Viktor Hamburger, W. M. Cowan, ed., pp. 74-133, Oxford University Press, New York.

Oppenheim, R. W., J. L. Maderdrut, and D. J. Wells (1982) Cell death of motorneurons in the chick embryo spinal cord. VI. Reduction of naturally occurring cell death in the thoracolumbar column of Terni by nerve growth factor. J. Comp. Neurol. 210: 174-189.

Pannese, E. (1963) Investigations on the ultrastructural changes of the spinal neurons in the course of axon regeneration and cell hypertrophy. II. Changes during cell hypertrophy and comparison between the ultrastructure of nerve cells of the same type under different functional conditions. Z. Zellforsch. $61: 561-586$.

Pannese, E. (1964) Number and structure of perisomatic satellite cells of spinal ganglia under normal conditions or during axon regeneration and neuronal hypertrophy. Z. Zellforsch 63: 568-592.

Pilar, G., L. Landmesser, and L. Burstein (1980) Competition for survival among developing ciliary ganglion cells. J. Neurophysiol. 43: $233-254$.

Pollack, E. D. (1969) Response of the lateral motor column to multiple forelimbs in Rana pipiens. Teratology 2: 159-162.

Scott, S. (1984) The effects of neural crest deletions on the development of sensory innervation patterns in embryonic chick hind limb. J. Physiol. (Lond.), in press.

Shoobridge, R., D. Velkou, and J. McCredie (1983) Neural crest ablation and limb morphogenesis. J. Exp. Zool. 225: 73-87.

Tosney, K. W. (1978) The early migration of neural crest cells in the trunk region of the avian embryo: An electron microscopic study. Dev. Biol. 62: 317-333.

Watkins, L. R., J. N. Johannessen, I. B. Kinsheck, and D. J. Mayer (1984) The neurochemical basis of footshock analgesia: The role of spinal cord serotin and norepinephrine. Brain Res. 290: 107-117.

Wishe, H. E., M. Roy, and S. J. Piliero (1980) A new organic solvent for use in the clearing of tissues. I. Soft tissue histology. Anat. Rec. 197: 283-288.

Yntema, C. L., and W. S. Hammond (1947) Depletions in the thoracolumbar sympathetic system following removal of neural crest in the chick. J. Comp. Neurol. 86: 237-266. 